\title{
HARMONIC SERIES WITH POLYLOGARITHMIC FUNCTIONS
}

Vuk Stojiljkovića ${ }^{a}$ Nicola Fabiano ${ }^{b}$, Vesna Šešum Čavićc

a University of Novi Sad, Faculty of Sciences, Novi Sad, Republic of Serbia, e-mail: vuk.stojiljkovic999@gmail.com, ORCID iD: @ https://orcid.org/0000-0002-4244-4342

b University of Belgrade, "Vinča" Institute of Nuclear Sciences Institute of National Importance for the Republic of Serbia, Belgrade, Republic of Serbia, e-mail: nicola.fabiano@gmail.com, corresponding author, ORCID iD: @ https://orcid.org/0000-0003-1645-2071

c TU Wien, Institute of Information Systems Engineering, Vienna, Republic of Austria; University of Belgrade, Faculty of Civil Engineering, Belgrade, Republic of Serbia, e-mail: vsesumcavic@grf.bg.ac.rs, ORCID iD: @https://orcid.org/0000-0002-0759-0686

DOI: 10.5937/vojtehg70-35148;https://doi.org/10.5937/vojtehg70-35148 FIELD: Mathematics ARTICLE TYPE: Original scientific paper

Abstract:

Introduction/purpose: Some sums of the polylogarithmic function associated with harmonic numbers are established.

Methods: The approach is based on using the summation methods.

Results: This paper generalizes the results of the zeta function series associated with the harmonic numbers.

Conclusions: Various interesting series as the consequence of the generalization are obtained.

Key words: polylogarithmic function, series, harmonic numbers, integration.

\section{Introduction and preliminaries}

The polylogarithm is a function in mathematics which was investigated intensively by many mathematicians. Many of them used different definitions but the one we use is the standard modern definition. For more information about the polylogarithm as a function consult the following book 
(Lewin, 1981). Questions about sums and their evaluations trace back to ancient times. Even the great Euler concerned himself with evaluating the $\zeta(2)$ known as the Basel problem, which was later generalized by him in view of finding a formula for even zeta values. More on various sums and evaluations can be found here (Hirschman, 2014; Knopp, 1990; Stojiljković, 2021; Davis, 2015). We will use the following notation throughout the paper. The first known definition is as follows.

DEFINITION 1. The polylogarithm, see (Lewin, 1981), is defined by a power series in $z$, given by

$$
\operatorname{Li}_{s}(z)=\sum_{k=1}^{+\infty} \frac{z^{k}}{k^{s}} .
$$

This definition is valid for the arbitrary complex order $s$ and for all complex arguments $z$ with $|z|<1$. We will also need the definition given by

$$
\operatorname{Li}_{s}(z)=\int_{0}^{z} \frac{\operatorname{Li}_{s-1}(z)}{z} d z .
$$

Also, the special case we will use frequently is

$$
\operatorname{Li}_{2}(z)=-\int_{0}^{z} \frac{\ln (1-z)}{z} d z .
$$

For $z=1$ we get the Riemann zeta function $\zeta$ which is also a function of the complex variable $s$. For more information see (Edwards, 1974; Fabiano, 2020).

$$
\mathrm{Li}_{s}(1)=\zeta(s)=\sum_{k=1}^{+\infty} \frac{1}{k^{s}}, \Re(s)>1
$$

The second definition is as follows.

DEFINITION 2. The harmonic numbers, see (Olaikhan, 2021), are defined as follows

$$
H_{n}:=1+\frac{1}{2}+\frac{1}{3}+\ldots+\frac{1}{n}
$$

for $n \geqslant 1$ and by definition $H_{0}=0$

The main results of this paper are the following. 
Theorem 1. Let $\operatorname{Li}_{s}(z)$ denote the polylogarithmic function. Then the following equality holds for $|z|<1$

$$
\begin{array}{r}
\sum_{k=1}^{+\infty} H_{k}\left(\operatorname{Li}_{s}(z)-z-\cdots-\frac{z^{k}}{k^{s}}\right)= \\
\int_{0}^{1} \frac{\operatorname{Li}_{s-1}(z)-\operatorname{Li}_{s-1}(z m)+z m-z}{1-m} d m \\
-\operatorname{Li}_{s-1}(z)+z .
\end{array}
$$

Theorem 2. Let $\operatorname{Li}_{s}(z)$ denote the polylogarithmic function. Then the following equality holds for $|z|<1$

$$
\begin{array}{r}
\sum_{k=1}^{+\infty} k H_{k}\left(\operatorname{Li}_{s}(z)-z-\frac{z^{2}}{2^{s}}-\cdots-\frac{z^{k}}{k^{s}}\right)= \\
\frac{1}{4}\left(\operatorname{Li}_{s-1}(z)-\operatorname{Li}_{s-2}(z)\right)+ \\
\frac{1}{2} \int_{0}^{1} \frac{\operatorname{Li}_{s-2}(z)+\operatorname{Li}_{s-1}(z m)-\operatorname{Li}_{s-1}(z)-\mathrm{Li}_{s-2}(z m)}{1-m} d m .
\end{array}
$$

Theorem 3. Let $\operatorname{Li}_{s}(z)$ denote the polylogarithmic function. Then the following equality holds for $|z|<1$

$$
\begin{array}{r}
\sum_{k=1}^{+\infty} H_{k}^{2}\left(\operatorname{Li}_{s}(z)-z-\frac{z^{2}}{2^{s}}-\ldots-\frac{z^{k}}{k^{s}}\right)= \\
\int_{0}^{1} \frac{\int_{0}^{1} \frac{\operatorname{Li}_{(s-1)}(z)-z-\left(\operatorname{Li}_{(s-1)}(z t)-z t\right)}{1-t} d t-\int_{0}^{1} \frac{\operatorname{Li}_{(s-1)}(z m)-z m-\left(\operatorname{Li}_{s-1)}(z m r)-z m r\right)}{1-r} d r}{(1-m)^{2}} d m \\
-\int_{0}^{1} \frac{\operatorname{Li}_{(s)}(z)-z-\left(\operatorname{Li}_{(s)}(z m)-z m\right)}{1-m} d m \\
-2\left(\int_{0}^{1} \frac{\operatorname{Li}_{s-1}(z)-\operatorname{Li}_{s-1}(z m)+z m-z}{1-m} d m-\operatorname{Li}_{s-1}(z)+z\right) .
\end{array}
$$

The corollaries of the results are given as follows.

COROLLARY 1. The following equalities come from theorem 1.

$$
\text { a) } \sum_{k=1}^{+\infty} H_{k}\left(\operatorname{Li}_{1}(z)-z-\ldots-\frac{z^{k}}{k^{1}}\right)=\frac{z+\ln (1-z)}{z-1},|z|<1
$$


Setting $z=\frac{1}{2}$ we get

$$
\text { b) } \sum_{k=1}^{+\infty} H_{k}\left(\operatorname{Li}_{1}\left(\frac{1}{2}\right)-\frac{1}{2}-\ldots-\frac{\left(\frac{1}{2}\right)^{k}}{k^{1}}\right)=\ln (4)-1 .
$$

We can also derive

$$
\text { c) } \sum_{k=1}^{+\infty} H_{k}\left(\operatorname{Li}_{2}(z)-z-\ldots-\frac{z^{k}}{k^{2}}\right)=-\operatorname{Li}_{2}\left(\frac{z}{z-1}\right)-\operatorname{Li}_{1}(z) .
$$

By setting $z=\frac{1}{2}$ we get

$$
\text { d) } \sum_{k=1}^{+\infty} H_{k}\left(\operatorname{Li}_{2}\left(\frac{1}{2}\right)-\frac{1}{2}-\ldots-\frac{\left(\frac{1}{2}\right)^{k}}{k^{2}}\right)=\frac{\pi^{2}}{12}-\ln (2) .
$$

COROLLARY 2. The following equalities come from Theorem 2.

$$
\begin{array}{r}
\text { a) } \begin{array}{r}
+\infty \\
k=1
\end{array} H_{k}\left(\operatorname{Li}_{2}(z)-z-\frac{(z)^{2}}{2^{2}}-\cdots-\frac{(z)^{k}}{k^{2}}\right)=\frac{1}{2} \frac{\ln (1-z)}{z-1}+ \\
\frac{1}{2} \operatorname{Li}_{2}\left(\frac{z}{z-1}\right)+\frac{1}{4}\left(\operatorname{Li}_{1}(z)-\operatorname{Li}_{0}(z)\right) .
\end{array}
$$

By setting $z=\frac{1}{2}$ we get

$$
\text { b) } \sum_{k=1}^{+\infty} k H_{k}\left(\operatorname{Li}_{2}\left(\frac{1}{2}\right)-\frac{1}{2}-\frac{\left(\frac{1}{2}\right)^{2}}{2^{2}}-\cdots-\frac{\left(\frac{1}{2}\right)^{k}}{k^{2}}\right)=\frac{5 \ln (2)}{4}-\frac{1}{4}-\frac{\pi^{2}}{24} .
$$

COROLLARY 3. The following equalities come from Theorem 3.

$$
\begin{gathered}
\text { a) } \sum_{k=1}^{+\infty} H_{k}^{2}\left(\operatorname{Li}_{1}(z)-z-\frac{z^{2}}{2^{1}}-\ldots-\frac{z^{k}}{k}\right)= \\
-\frac{4 z+\ln (1-z)(4+\ln (1-z))-2 z \operatorname{Li}_{2}\left(\frac{z}{z-1}\right)}{2(z-1)} .
\end{gathered}
$$

By setting $z=\frac{1}{2}$ we get

b) $\sum_{k=1}^{+\infty} H_{k}^{2}\left(\operatorname{Li}_{(1)}\left(\frac{1}{2}\right)-\frac{1}{2}-\frac{\left(\frac{1}{2}\right)^{2}}{2}-\ldots-\frac{\left(\frac{1}{2}\right)^{k}}{k}\right)=2+\frac{\pi^{2}}{12}+\ln ^{2}(2)-\ln (16)$. 


\section{Main results}

We will need some lemmas in order to proceed further. The following lemma will be extensively used throughout the paper.

Lemma 1. The following equality holds for $|z|<1$.

$$
\sum_{k=1}^{+\infty} \frac{z^{k+1}}{(k+1)^{s-1}}=\operatorname{Li}_{s-1}(z)-z
$$

Proof. Follows from the definition of the polylogarithm.

We will need the following in order to proceed further.

We will need in our analysis Abel's summation formula (Bonar \& Koury, 2006, p.55),(Lewin, 1981, p.258), which states that if $\left(a_{n}\right)_{n \geqslant 1}$ and $\left(b_{n}\right)_{n \geqslant 1}$ are two sequences of real numbers and $A_{n}=\sum_{k=1}^{n} a_{k}$, then

$$
\sum_{k=1}^{n} a_{k} b_{k}=A_{n} b_{n+1}+\sum_{k=1}^{n} A_{k}\left(b_{k}-b_{k+1}\right)
$$

We will also be using, in our calculations, the infinite version of the preceding formula

$$
\sum_{k=1}^{+\infty} a_{k} b_{k}=\lim _{n \rightarrow+\infty}\left(A_{n} b_{n+1}\right)+\sum_{k=1}^{+\infty} A_{k}\left(b_{k}-b_{k+1}\right) .
$$

The second lemma will be given.

LEMMA 2. The following identity holds:

$$
\sum_{k=1}^{n} H_{k}=(n+1) H_{n+1}-(n+1)
$$

Proof. We will prove it using the Abel's summation (finite version). By choosing $a_{k}=1, b_{k}=H_{k}$ we get 


$$
\begin{aligned}
\sum_{k=1}^{n} H_{k} & =n H_{n+1}+\sum_{k=1}^{n} k \cdot\left(-\frac{1}{k+1}\right)=n H_{n+1}-\sum_{k=1}^{n} \frac{k}{k+1}=n H_{n+1}-n \\
& +\sum_{k=1}^{n} \frac{1}{k+1}=n H_{n+1}-n+\left(H_{n+1}-1\right)=(n+1) H_{n+1}-(n+1)
\end{aligned}
$$

and the proof is done.

The third lemma that we will need.

LeMmA 3. The following equality holds.

$$
\sum_{k=1}^{n} k H_{k}=\frac{n(n+1)}{2} H_{n+1}-\frac{n(n+1)}{4}
$$

Proof. We will prove it using the Abel's summation (finite version). By choosing $a_{k}=k, b_{k}=H_{k}$ we get

$$
\begin{aligned}
& \sum_{k=1}^{n} k H_{k}=\frac{n(n+1)}{2} H_{n+1}+\sum_{k=1}^{n} \frac{k(k+1)}{2}\left(-\frac{1}{k+1}\right)= \\
& =\frac{n(n+1)}{2} H_{n+1}-\sum_{k=1}^{n} \frac{k}{2}=\frac{n(n+1)}{2} H_{n+1}-\frac{n(n+1)}{4}
\end{aligned}
$$

and the proof is complete.

LEMMA 4. The following equality holds for any $q$ and for $|z|<1$

$$
\lim _{k \rightarrow+\infty} k^{q}\left(\operatorname{Li}_{s}(z)-z-\cdots-\frac{z^{k+1}}{(k+1)^{s}}\right)=0 .
$$

Proof. Let us observe the expression inside the brackets

$$
\left(\operatorname{Li}_{s}(z)-z-\cdots-\frac{z^{k+1}}{(k+1)^{s}}\right)=\frac{z^{k+2}}{(k+2)^{s}}+\frac{z^{k+3}}{(k+3)^{s}}+\frac{z^{k+4}}{(k+4)^{s}}+\ldots
$$


What we can realise is that every term is less than $\frac{z^{k}}{k^{s}}$; therefore, by

$$
\frac{z^{k+2}}{(k+2)^{s}}+\frac{z^{k+3}}{(k+3)^{s}}+\frac{z^{k+4}}{(k+4)^{s}}+\ldots \leq \frac{z^{k}}{k^{s}}+\frac{z^{k}}{k^{s}}+\ldots
$$

multiplying both sides by $k^{q}$ and letting the limit go to infinity, we get

$$
\lim _{k \rightarrow+\infty} k^{q}\left(\operatorname{Li}_{s}(z)-z-\ldots .-\frac{z^{k+1}}{(k+1)^{s}}\right) \leq \lim _{k \rightarrow+\infty} k^{q} \cdot \frac{k z^{k}}{k^{s}}=\lim _{k \rightarrow+\infty} \frac{z^{k}}{k^{s-q-1}}
$$

and this will go to zero independently of $s-q-1$ because $|z|<1$ and $z^{k}$ goes faster to zero than any power of the form $k^{s-q-1}$.

The proof is complete.

We give our first generalization of the zeta function series.

LEMmA 5. Let $\mathrm{Li}_{s}(z)$ denote the polylogarithmic function. Then the following equality holds for $|z|<1$

$$
\sum_{k=1}^{+\infty}\left(\operatorname{Li}_{s}(z)-z-\frac{z^{2}}{2^{s}}-\ldots-\frac{z^{k}}{k^{s}}\right)=\operatorname{Li}_{s-1}(z)-\operatorname{Li}_{s}(z) .
$$

Proof. We apply the Abel's summation formula with $a_{k}=1$ and $b_{k}=$ $\mathrm{Li}_{s}(z)-z-\frac{z^{2}}{2^{s}}-\ldots-\frac{z^{k}}{k^{s}}$ from which we get

$$
\begin{gathered}
\sum_{k=1}^{+\infty}\left(\operatorname{Li}_{s}(z)-z-\frac{z^{2}}{2^{s}}-\ldots-\frac{z^{k}}{k^{s}}\right)= \\
=\lim _{k \rightarrow+\infty} k\left(\operatorname{Li}_{s}(z)-z-\frac{z^{2}}{2^{s}}-\ldots-\frac{z^{k}}{k^{s}}-\frac{z^{k+1}}{(k+1)^{s}}\right)+\sum_{k=1}^{+\infty} \frac{k z^{k+1}}{(k+1)^{s}} .
\end{gathered}
$$

Since the first term goes to zero when $k \rightarrow+\infty$, Lemma $4(q=1)$, we get 


$$
\sum_{k=1}^{+\infty}\left(\operatorname{Li}_{s}(z)-z-\frac{z^{2}}{2^{s}}-\ldots-\frac{z^{k}}{k^{s}}\right)=\sum_{k=1}^{+\infty} \frac{k z^{k+1}}{(k+1)^{s}} .
$$

Adding and subtracting 1 in the numerator leaves us with two sums

$$
\sum_{k=1}^{+\infty} \frac{z^{k+1}}{(k+1)^{s-1}}-\sum_{k=1}^{+\infty} \frac{z^{k+1}}{(k+1)^{s}}=\operatorname{Li}_{s-1}(z)-\operatorname{Li}_{s}(z)
$$

because of Lemma 1. The proof is complete.

In the following we give a proof of Theorem 1.

THEOREM 1. Let $\operatorname{Li}_{s}(z)$ denote the polylogarithmic function. Then the following equality holds for $|z|<1$

$$
\begin{array}{r}
\sum_{k=1}^{+\infty} H_{k}\left(\operatorname{Li}_{s}(z)-z-\cdots-\frac{z^{k}}{k^{s}}\right)=\int_{0}^{1} \frac{\mathrm{Li}_{s-1}(z)-\mathrm{Li}_{s-1}(z m)+z m-z}{1-m} d m \\
-\mathrm{Li}_{s-1}(z)+z .
\end{array}
$$

Proof. By using Abel's theorem (infinite version) and choosing $a_{k}=H_{k}, b_{k}=\operatorname{Li}_{s}(z)-z-\cdots-\frac{z^{k}}{k^{s}}$ and Lemma 2 we get

$$
\begin{gathered}
\sum_{k=1}^{+\infty} H_{k}\left(\operatorname{Li}_{s}(z)-z-\ldots-\frac{z^{k}}{k^{s}}\right)= \\
=\lim _{k \rightarrow+\infty}\left((k+1) H_{k+1}-(k+1)\right)\left(\operatorname{Li}_{s}(z)-z-\ldots-\frac{z^{k}}{k^{s}}-\frac{z^{k+1}}{(k+1)^{s}}\right)+ \\
+\sum_{k=1}^{+\infty} \frac{\left((k+1) H_{k+1}-(k+1)\right) z^{k+1}}{(k+1)^{s}} .
\end{gathered}
$$

Since the first term goes to zero when $k \rightarrow+\infty$, Lemma $4(q=2)$, the above equals to:

$$
\sum_{k=1}^{+\infty} \frac{\left((k+1) H_{k+1}-(k+1)\right) z^{k+1}}{(k+1)^{s}}=\sum_{k=1}^{+\infty} \frac{H_{k+1} z^{k+1}}{(k+1)^{s-1}}-\sum_{k=1}^{+\infty} \frac{z^{k+1}}{(k+1)^{s-1}} .
$$

The second sum follows from Lemma 1. In the first sum we will rewrite the harmonic number as an integral and interchange the sum and the inte- 
gral thanks to Fubini's theorem:

$$
\begin{gathered}
\sum_{k=1}^{+\infty} \frac{H_{k+1} z^{k+1}}{(k+1)^{s-1}}=\sum_{k=1}^{+\infty} \frac{z^{k+1}}{(k+1)^{s-1}} \int_{0}^{1} \frac{1-m^{k+1}}{1-m} d m \\
=\int_{0}^{1} \sum_{k=1}^{+\infty} \frac{z^{k+1}}{(k+1)^{s-1}} \frac{1-m^{k+1}}{1-m} d m .
\end{gathered}
$$

By rewriting it as two sums, we get

$$
\int_{0}^{1} \frac{\sum_{k=1}^{+\infty} \frac{z^{k+1}}{(k+1)^{s-1}}-\sum_{k=1}^{+\infty} \frac{(m z)^{k+1}}{(k+1)^{s-1}}}{1-m} d m .
$$

Using the results from the Lemma 1 leaves us with

$$
=\int_{0}^{1} \frac{\operatorname{Li}_{s-1}(z)-z-\left(\operatorname{Li}_{s-1}(z m)-z m\right)}{1-m} d m .
$$

Which, when substituted above, gives us:

$$
\begin{array}{r}
\sum_{k=1}^{+\infty} H_{k}\left(\operatorname{Li}_{s}(z)-z-\ldots-\frac{z^{k}}{k^{s}}\right)=\int_{0}^{1} \frac{\operatorname{Li}_{s-1}(z)-\mathrm{Li}_{s-1}(z m)+z m-z}{1-m} d m \\
-\mathrm{Li}_{s-1}(z)+z
\end{array}
$$

Now we prove Corollary 1 , part a).

When $s=1$ it can be shown, after a long and tedious calculation, that the following holds

$$
\sum_{k=1}^{+\infty} H_{k}\left(\operatorname{Li}_{1}(z)-z-\ldots-\frac{z^{k}}{k^{1}}\right)=\frac{z+\ln (1-z)}{z-1},|z|<1 .
$$

By setting $z=\frac{1}{2}$ we get b)

$$
\sum_{k=1}^{+\infty} H_{k}\left(\operatorname{Li}_{1}\left(\frac{1}{2}\right)-\frac{1}{2}-\ldots-\frac{\left(\frac{1}{2}\right)^{k}}{k^{1}}\right)=\ln (4)-1 .
$$


When $s=2$ it can be shown, similarly to the case $s=1$, that c) part holds

$$
\sum_{k=1}^{+\infty} H_{k}\left(\operatorname{Li}_{2}(z)-z-\ldots-\frac{z^{k}}{k^{2}}\right)=-\operatorname{Li}_{2}\left(\frac{z}{z-1}\right)-\operatorname{Li}_{1}(z) .
$$

By setting $z=\frac{1}{2}$ we arrive at d)

$$
\sum_{k=1}^{+\infty} H_{k}\left(\operatorname{Li}_{2}\left(\frac{1}{2}\right)-\frac{1}{2}-\ldots-\frac{\left(\frac{1}{2}\right)^{k}}{k^{2}}\right)=\frac{\pi^{2}}{12}-\ln (2) .
$$

In the following we give proof of Theorem 2.

THEOREM 2. Let $\operatorname{Li}_{s}(z)$ denote the polylogarithmic function. Then the following equality holds for $|z|<1$

$$
\begin{aligned}
\sum_{k=1}^{+\infty} k H_{k} & \left(\operatorname{Li}_{s}(z)-z-\frac{z^{2}}{2^{s}}-\ldots-\frac{z^{k}}{k^{s}}\right)=\frac{1}{4}\left(\operatorname{Li}_{s-1}(z)-\mathrm{Li}_{s-2}(z)\right)+ \\
& \frac{1}{2} \int_{0}^{1} \frac{\mathrm{Li}_{s-2}(z)+\mathrm{Li}_{s-1}(z m)-\mathrm{Li}_{s-1}(z)-\mathrm{Li}_{s-2}(z m)}{1-m} d m .
\end{aligned}
$$

Proof. Using the Abel's summation with $a_{k}=k H_{k}$ and $b_{k}=\mathrm{Li}_{s}(z)-\frac{z}{1^{s}}-$ $\ldots-\frac{z^{k}}{k^{s}}$ and Lemma 3 for the $a_{k}$ part gives

$$
\begin{aligned}
\sum_{k=1}^{+\infty} k H_{k}\left(\operatorname{Li}_{s}(z)-z-\frac{z^{2}}{2^{s}}-\ldots-\frac{z^{k}}{k^{s}}\right)=\lim _{k \rightarrow+\infty}\left(\frac{k(k+1) H_{k+1}}{2}-\frac{k(k+1)}{4}\right) \\
\cdot\left(\operatorname{Li}_{s}(z)-z-\frac{z^{2}}{2^{s}}-\ldots-\frac{z^{k}}{k^{s}}-\frac{z^{k+1}}{(k+1)^{s}}\right)+ \\
\sum_{k=1}^{+\infty}\left(\frac{k(k+1) H_{k+1}}{2}-\frac{k(k+1)}{4}\right) \cdot \frac{z^{k+1}}{(k+1)^{s}} .
\end{aligned}
$$

The expression in the brackets goes to zero by Lemma 4, so we are left with:

$$
\frac{1}{2} \sum_{k=1}^{+\infty} \frac{k H_{k+1} z^{k+1}}{(k+1)^{s-1}}-\frac{1}{4} \sum_{k=1}^{+\infty} \frac{k z^{k+1}}{(k+1)^{s-1}}
$$

We will use Lemma 1:

$$
\sum_{k=1}^{+\infty} \frac{k z^{k+1}}{(k+1)^{s}}=\mathrm{Li}_{s-1}(z)-\mathrm{Li}_{s}(z) .
$$


As we can see, the second sum is the expression above with $s$ shifted by -1 and multiplied by $\frac{1}{4}$. For the first sum, we will rewrite the harmonic number into its integral form.

$$
\begin{aligned}
& \frac{1}{2} \sum_{k=1}^{+\infty} \frac{k H_{k+1} z^{k+1}}{(k+1)^{s-1}}=\frac{1}{2} \sum_{k=1}^{+\infty} \frac{k z^{k+1}}{(k+1)^{s-1}} \int_{0}^{1} \frac{1-m^{k+1}}{1-m} d m= \\
& \int_{0}^{1} \frac{1}{2} \sum_{k=1}^{+\infty} \frac{k z^{k+1}}{(k+1)^{s-1}} \frac{1-m^{k+1}}{1-m} d m= \\
& \frac{1}{2} \int_{0}^{1} \frac{\sum_{k=1}^{+\infty} \frac{k z^{k+1}}{(k+1)^{s-1}}-\sum_{k=1}^{+\infty} \frac{k(z m)^{k+1}}{(k+1)^{s-1}}}{1-m} d m .
\end{aligned}
$$

Both sums are of the form given above. Therefore, we get

$$
\frac{1}{2} \int_{0}^{1} \frac{\mathrm{Li}_{s-2}(z)-\mathrm{Li}_{s-1}(z)-\left(\mathrm{Li}_{s-2}(z m)-\mathrm{Li}_{s-1}(z m)\right)}{1-m} d m .
$$

By incorporating this into the original equality, we get

$$
\begin{array}{r}
\sum_{k=1}^{+\infty} k H_{k}\left(\operatorname{Li}_{s}(z)-z-\frac{z^{2}}{2^{s}}-\ldots-\frac{z^{k}}{k^{s}}\right)=\frac{1}{4}\left(\operatorname{Li}_{s-1}(z)-\mathrm{Li}_{s-2}(z)\right)+ \\
\frac{1}{2} \int_{0}^{1} \frac{\mathrm{Li}_{s-2}(z)+\mathrm{Li}_{s-1}(z m)-\mathrm{Li}_{s-1}(z)-\mathrm{Li}_{s-2}(z m)}{1-m} d m .
\end{array}
$$

By setting $s=2$ it can be shown that Corollary 2 part a) holds

$$
\begin{array}{r}
\sum_{k=1}^{+\infty} k H_{k}\left(\operatorname{Li}_{2}(z)-z-\frac{(z)^{2}}{2^{2}}-\ldots-\frac{(z)^{k}}{k^{2}}\right)=\frac{1}{2} \frac{\ln (1-z)}{z-1}+\frac{1}{2} \operatorname{Li}_{2}\left(\frac{z}{z-1}\right)+ \\
\frac{1}{4}\left(\operatorname{Li}_{1}(z)-\operatorname{Li}_{0}(z)\right) .
\end{array}
$$

By setting $z=\frac{1}{2}$ we arrive at part b)

$$
\sum_{k=1}^{+\infty} k H_{k}\left(\operatorname{Li}_{2}\left(\frac{1}{2}\right)-\frac{1}{2}-\frac{\left(\frac{1}{2}\right)^{2}}{2^{2}}-\ldots-\frac{\left(\frac{1}{2}\right)^{k}}{k^{2}}\right)=\frac{1}{2}\left(-\frac{\pi^{2}}{12}+\ln (4)\right)+
$$




$$
\frac{1}{4}(\ln (2)-1)
$$

Our significant result in this paper is given in the following theorem. The next theorem will use all the previous results.

THEOREM 3. Let $\operatorname{Li}_{s}(z)$ denote the polylogarithmic function. Then the foIlowing equality holds for $|z|<1$

$$
\begin{array}{r}
\sum_{k=1}^{+\infty} H_{k}^{2}\left(\operatorname{Li}_{s}(z)-z-\frac{z^{2}}{2^{s}}-\ldots-\frac{z^{k}}{k^{s}}\right)= \\
\int_{0}^{1} \frac{\int_{0}^{1} \frac{\mathrm{Li}_{(s-1)}(z)-z-\left(\mathrm{Li}_{(s-1)}(z t)-z t\right)}{1-t} d t-\int_{0}^{1} \frac{\mathrm{Li}_{(s-1)}(z m)-z m-\left(\mathrm{Li}_{s-1)}(z m r)-z m r\right)}{1-r} d r}{1-m} d m \\
-\int_{0}^{1} \frac{\mathrm{Li}_{(s)}(z)-z-\left(\mathrm{Li}_{(s)}(z m)-z m\right)}{1-m} d m- \\
2\left(\int_{0}^{1} \frac{\mathrm{Li}_{s-1}(z)-\mathrm{Li}_{s-1}(z m)+z m-z}{1-m} d m-\mathrm{Li}_{s-1}(z)+z\right) .
\end{array}
$$

Proof. We will use Abel's summation method, choosing $a_{k}=H_{k}, b_{k}=H_{k}\left(\operatorname{Li}_{s}(z)-z-\frac{z^{2}}{2^{s}}-\ldots-\frac{z^{k}}{k^{s}}\right)$ with Lemma 2, we will use the following notation to minimize the clutter in the formulas, let us call $S_{k}=\operatorname{Li}_{s}(z)-z-\frac{z^{2}}{2^{s}}-\ldots-\frac{z^{k}}{k^{s}}$. By evaluating $b_{k}-b_{k+1}$ we get

$$
b_{k}-b_{k+1}=\frac{H_{k} z^{k+1}}{(k+1)^{s}}-\frac{S_{k}}{(k+1)}+\frac{z^{k+1}}{(k+1)^{s+1}} .
$$

By using Abel's summation we get

$$
\begin{gathered}
\lim _{k \rightarrow+\infty}\left((k+1) H_{k+1}-(k+1)\right) H_{k+1} S_{k+1}+ \\
+\sum_{k=1}^{+\infty}\left((k+1) H_{k+1}-(k+1)\right)\left(\frac{H_{k} z^{k+1}}{(k+1)^{s}}-\frac{S_{k}}{(k+1)}+\frac{z^{k+1}}{(k+1)^{s+1}}\right) .
\end{gathered}
$$

The expression in the limit goes to zero by Lemma 4 . We are left with the sum

$$
\sum_{k=1}^{+\infty} \frac{H_{k+1} H_{k} z^{k+1}}{(k+1)^{s-1}}-\sum_{k=1}^{+\infty} S_{k} H_{k+1}+\sum_{k=1}^{+\infty} \frac{z^{k+1} H_{k+1}}{(k+1)^{s}}-\sum_{k=1}^{+\infty} \frac{H_{k} z^{k+1}}{(k+1)^{s-1}}+
$$




$$
\sum_{k=1}^{+\infty} S_{k}-\sum_{k=1}^{+\infty} \frac{z^{k+1}}{(k+1)^{s}}
$$

We know the third term from the proof of Theorem 1, the fifth term from Lemma 5 and the sixth term from Lemma 1. Let us focus on the second one, $\sum_{k=1}^{+\infty} H_{k+1} S_{k}$. This is a separate problem we must deal with. So let us write

$$
\sum_{k=1}^{+\infty} H_{k+1} S_{k}=\sum_{k=1}^{+\infty}\left(H_{k}+\frac{1}{k+1}\right) S_{k}=\sum_{k=1}^{+\infty} H_{k} S_{k}+\sum_{k=1}^{+\infty} \frac{S_{k}}{k+1} .
$$

The first term is known from Theorem 1, but the second one is not, so we will use again Abel's summation method choosing $a_{k}=\frac{1}{k+1}, b_{k}=S_{k}$. We get

$\lim _{k \rightarrow+\infty}\left(H_{k+1}-1\right) S_{k+1}+\sum_{k=1}^{+\infty}\left(H_{k+1}-1\right) \frac{z^{k+1}}{(k+1)^{s}}=\sum_{k=1}^{+\infty} \frac{z^{k+1} H_{k+1}}{(k+1)^{s}}-\sum_{k=1}^{+\infty} \frac{z^{k+1}}{(k+1)^{s}}$

The first sum is from the proof of Theorem 1 while the second one is from Lemma 1; therefore, the original second sum is done. Let us deal with the fourth sum:

$$
\begin{array}{r}
\sum_{k=1}^{+\infty} \frac{H_{k} z^{k+1}}{(k+1)^{s-1}}=\sum_{k=1}^{+\infty}\left(H_{k}+\frac{1}{k+1}-\frac{1}{k+1}\right) \frac{z^{k+1}}{(k+1)^{s-1}}= \\
\sum_{k=1}^{+\infty} \frac{H_{k+1} z^{k+1}}{(k+1)^{s-1}}-\sum_{k=1}^{+\infty} \frac{z^{k+1}}{(k+1)^{s}}
\end{array}
$$

The first sum is from the proof of Theorem 1 while the second one is from Lemma 1. Therefore, the fourth sum is done. Let us focus on the first one.

$$
\begin{aligned}
\sum_{k=1}^{+\infty} \frac{H_{k+1} H_{k} z^{k+1}}{(k+1)^{s-1}}= & \sum_{k=1}^{+\infty} \frac{H_{k+1}\left(H_{k}+\frac{1}{k+1}-\frac{1}{k+1}\right)}{(k+1)^{s-1}}= \\
& \sum_{k=1}^{+\infty} \frac{H_{k+1}^{2} z^{k+1}}{(k+1)^{s-1}}-\sum_{k=1}^{+\infty} \frac{z^{k+1} H_{k+1}}{(k+1)^{s}}
\end{aligned}
$$


The second one is from the proof of Theorem 1, but we need to dig further for the first one

$$
\begin{array}{r}
\sum_{k=1}^{+\infty} \frac{H_{k+1}^{2} z^{k+1}}{(k+1)^{s-1}}=\sum_{k=1}^{+\infty} \frac{H_{k+1} z^{k+1}}{(k+1)^{s-1}} \int_{0}^{1} \frac{1-m^{k+1}}{1-m} d m= \\
\int_{0}^{1} \frac{\sum_{k=1}^{+\infty} \frac{H_{k+1} z^{k+1}}{(k+1)^{s-1}}-\sum_{k=1}^{+\infty} \frac{H_{k+1}(m z)^{k+1}}{(k+1)^{s-1}}}{1-m} d m .
\end{array}
$$

The second term is the same as in Theorem 1 when taking $z$ as $z m$; therefore, the result follows.

$$
\sum_{k=1}^{+\infty} \frac{H_{k+1}(z m)^{k+1}}{(k+1)^{s-1}}=\int_{0}^{1} \frac{\operatorname{Li}_{s-1}(z m)-z m-\left(\mathrm{Li}_{s-1}(z m r)-z m r\right)}{1-r} d r
$$

While the first one we have directly from the proof of Theorem 1

$$
\sum_{k=1}^{+\infty} \frac{H_{k+1} z^{k+1}}{(k+1)^{s-1}}=\int_{0}^{1} \frac{\operatorname{Li}_{(s-1)}(z)-z-\left(\operatorname{Li}_{(s-1)}(z t)-z t\right)}{1-t} d t .
$$

Therefore, by putting all together, we obtain

$$
\begin{array}{r}
\sum_{k=1}^{+\infty} \frac{H_{k+1} H_{k} z^{k+1}}{(k+1)^{s-1}}-\sum_{k=1}^{+\infty} S_{k} H_{k+1}+\sum_{k=1}^{+\infty} \frac{z^{k+1} H_{k+1}}{(k+1)^{s}}-\sum_{k=1}^{+\infty} \frac{H_{k} z^{k+1}}{(k+1)^{s-1}}+ \\
\sum_{k=1}^{+\infty} S_{k}-\sum_{k=1}^{+\infty} \frac{z^{k+1}}{(k+1)^{s}}=\sum_{k=1}^{+\infty} \frac{H_{k+1}^{2} z^{k+1}}{(k+1)^{s-1}}-\sum_{k=1}^{+\infty} \frac{z^{k+1} H_{k+1}}{(k+1)^{s}}-\left(\sum_{k=1}^{+\infty} H_{k} S_{k}+\right. \\
\left.\sum_{k=1}^{+\infty} \frac{z^{k+1} H_{k+1}}{(k+1)^{s}}-\sum_{k=1}^{+\infty} \frac{z^{k+1}}{(k+1)^{s}}\right)+\sum_{k=1}^{+\infty} \frac{z^{k+1} H_{k+1}}{(k+1)^{s}}-\left(\sum_{k=1}^{+\infty} \frac{H_{k+1} z^{k+1}}{(k+1)^{s-1}}-\right. \\
\left.\sum_{k=1}^{+\infty} \frac{z^{k+1}}{(k+1)^{s}}\right)+\sum_{k=1}^{+\infty} S_{k}-\sum_{k=1}^{+\infty} \frac{z^{k+1}}{(k+1)^{s}} .
\end{array}
$$

We can see that four of the terms will cancel themselves; then we plug the polylogarithm expressions we have got and establish the equality.

$$
\sum_{k=1}^{+\infty} H_{k}^{2}\left(\operatorname{Li}_{s}(z)-z-\frac{z^{2}}{2^{s}}-\ldots-\frac{z^{k}}{k^{s}}\right)=
$$




$$
\begin{aligned}
& \int_{0}^{1} \frac{\int_{0}^{1} \frac{\operatorname{Li}_{(s-1)}(z)-z-\left(\operatorname{Li}_{(s-1)}(z t)-z t\right)}{1-t} d t-\int_{0}^{1} \frac{\operatorname{Li}_{(s-1)}(z m)-z m-\left(\operatorname{Li}_{(s-1)}(z m r)-z m r\right)}{1-r} d r}{1-m} d m \\
& -\int_{0}^{1} \frac{\operatorname{Li}_{(s)}(z)-z-\left(\operatorname{Li}_{(s)}(z m)-z m\right)}{1-m} d m- \\
& 2\left(\int_{0}^{1} \frac{\mathrm{Li}_{s-1}(z)-\mathrm{Li}_{s-1}(z m)+z m-z}{1-m} d m-\mathrm{Li}_{s-1}(z)+z\right) \text {. }
\end{aligned}
$$

\section{Some examples of series}

The usage of the previously derived theorems will be displayed in the following examples. Equipped with the series in a closed form we have derived, we can get many series via incorporating the values from the domain which is $|z|<1$. By letting $z=\frac{\sqrt{5}-1}{2}$ in Corollary 1 part c), Corollary 2 part a) and Corollary 3 part a) we get, respectively

$$
\begin{gathered}
\sum_{k=1}^{+\infty} H_{k}\left(\operatorname{Li}_{2}\left(\frac{\sqrt{5}-1}{2}\right)-\frac{\sqrt{5}-1}{2}-\ldots-\frac{\left(\frac{\sqrt{5}-1}{2}\right)^{k}}{k^{2}}\right)= \\
=\frac{\pi^{2}}{10}+\ln ^{2}\left(\frac{1}{2}+\frac{\sqrt{5}}{2}\right)+\ln \left(\frac{1}{2}(3-\sqrt{5})\right) \\
\sum_{k=1}^{+\infty} k H_{k}\left(\operatorname{Li}_{2}\left(\frac{\sqrt{5}-1}{2}\right)-\frac{\sqrt{5}-1}{2}-\frac{\left(\frac{\sqrt{5}-1}{2}\right)^{2}}{2^{2}}-\ldots-\frac{\left(\frac{\sqrt{5}-1}{2}\right)^{k}}{k^{2}}\right)=
\end{gathered}
$$




$$
\begin{gathered}
=-\frac{1-\sqrt{5}+(\sqrt{5}-7) \ln \left(\frac{1}{2}(3-\sqrt{5})\right)+2(\sqrt{5}-3) \operatorname{csch}^{-1}(2)^{2}}{4(\sqrt{5}-3)}-\frac{\pi^{2}}{20} \\
\sum_{k=1}^{+\infty} H_{k}^{2}\left(\operatorname{Li}_{1}\left(\frac{\sqrt{5}-1}{2}\right)-\frac{\sqrt{5}-1}{2}-\frac{\left(\frac{\sqrt{5}-1}{2}\right)^{2}}{2^{1}}-\ldots-\frac{\left(\frac{\sqrt{5}-1}{2}\right)^{k}}{k}\right)= \\
=-\frac{1}{10(\sqrt{5}-3)}\left((\sqrt{5}-1) \pi^{2}-20+20 \sqrt{5}\right)+ \\
+\frac{-1}{\sqrt{5}-3}\left(\ln \left(\frac{1}{2}(47-21 \sqrt{5})\right)+\cosh ^{-1}\left(\frac{3}{2}\right)^{2}+(\sqrt{5}-1) \operatorname{csch}^{-1}(2)^{2}\right)
\end{gathered}
$$

More interesting sums can be obtained incorporating in the value $z=$ $\frac{3-\sqrt{5}}{2}$.

By setting $z=\frac{3-\sqrt{5}}{2}$ in Corollary 1 part c), Corollary 2 part a) and Corollary 3 part a), we get, respectively

$$
\begin{gathered}
\sum_{k=1}^{+\infty} H_{k}\left(\operatorname{Li}_{2}\left(\frac{3-\sqrt{5}}{2}\right)-\frac{3-\sqrt{5}}{2}-\ldots-\frac{\left(\frac{3-\sqrt{5}}{2}\right)^{k}}{k^{2}}\right)= \\
=\frac{\pi^{2}}{15}-\frac{1}{2} \operatorname{csch}^{-1}(2)\left(2+\operatorname{csch}^{-1}(2)\right) \\
\sum_{k=1}^{+\infty} k H_{k}\left(\operatorname{Li}_{2}\left(\frac{3-\sqrt{5}}{2}\right)-\frac{3-\sqrt{5}}{2}-\ldots-\frac{\left(\frac{3-\sqrt{5}}{2}\right)^{k}}{k^{2}}\right)=
\end{gathered}
$$

$\frac{1}{16}\left(2-2 \sqrt{5}+(1+\sqrt{5}) \sinh ^{-1}(2)+\operatorname{csch}^{-1}(2)\left(5+\sqrt{5}+4 \operatorname{csch}^{-1}(2)\right)\right)-\frac{\pi^{2}}{30}$

$$
\sum_{k=1}^{+\infty} H_{k}^{2}\left(\operatorname{Li}_{2}\left(\frac{3-\sqrt{5}}{2}\right)-\frac{3-\sqrt{5}}{2}-\ldots-\frac{\left(\frac{3-\sqrt{5}}{2}\right)^{k}}{k^{2}}\right)=
$$

$=\frac{1}{30}\left((\sqrt{5}-1) \pi^{2}+15\left(2(\sqrt{5}-1)-2(1+\sqrt{5}) \operatorname{csch}^{-1}(2)+\operatorname{csch}^{-1}(2)^{2}\right)\right)$.

The numerical values of $\mathrm{Li}_{2}$ at the points $z=\frac{\sqrt{5}-1}{2},-\frac{\sqrt{5}-1}{2},-\frac{1+\sqrt{5}}{2}$ can be found here (Lewin, 1981). Many more series can be obtained by substituting different values. 


\section{Conclusions}

1. To assure the accuracy of the results, we verified all the numerical series identities through Wolfram Alpha.

2. Further questions can be asked regarding the sums with harmonic numbers of an arbitrary order as to, whether it is possible to find more of them of the form $H_{\frac{n}{k}}$ for some fixed $k$.

3. In this paper, we generalized the results given in (Furdui, 2016) as the polylogarithm is a generalization of the zeta function since $\operatorname{Li}_{s}(1)=\zeta(s)$. We can obtain many more series by varying the two parameters $z$ and $s$.

\section{References}

Bonar, D.D. and Khoury, M.J. 2006. Real Infinite Series. Washington D.C., American Mathematical Society: MAA Press. ISBN: 978-1-4704-4782-3.

Davis, H.T. 2015. The Summation of Series (Dover Books on Mathematics). Mineola, New York: Dover Publications. ISBN-13: 978-0486789682.

Edwards, M.H. 1974. Riemann's Zeta Function. Mineola, New York: Dover Publications. ISBN-13: 978-0486417400.

Fabiano, N. 2020. Zeta function and some of its properties. Vojnotehnički glasnik/Military Technical Courier, 68(4), pp.895-906. Available at: https://doi.org/10.5937/vojtehg68-28535.

Furdui, O. 2016. Harmonic series with polygamma functions. Journal of Classical Analysis, 8(2), pp.123-130. Available at: https://doi.org/10.7153/jca-08-11.

Hirschman, I.I. 2014. Infinite series (Dover Books on Mathematics). Mineola, New York: Dover Publications. ISBN-13: 978-0-486-78975-0.

Knopp, K. 1990. Theory and Applications of Infinite Series. Mineola, New York: Dover Publications. ISBN-13: 978-0-486-66165-2.

Lewin, L. 1981. Polylogarithms and associated functions. Elsevier Science Ltd. ISBN-13: 978-0444005502.

Olaikhan, A.S. 2021. An Introduction To The Harmonic Series And Logarithmic Integrals: For High School Students Up To Researcher. Ali Shadhar Olaikhan (private edition). ISBN-13: 978-1-7367360-0-5.

Stojiljković, V. 2021. Some Series Associated with Central Binomial Coefficients and Harmonic Numbers. Octogon Mathematical Magazine, 29(2). 


\section{ГАРМОНИЧЕСКИЙ РЯД С ПОЛИЛОГАРИФМИЧЕСКИМИ} ФУНКЦИЯМИ

Вук Стоилькович ${ }^{a}$, Никола Фабиано ${ }^{\circ}$, Весна Шешум Чавич

а Нови-Садский университет, фракультет естественных наук, г. Нови-Сад, Республика Сербия

${ }^{\sigma}$ Белградский университет, Институт ядерных исследований «Винча» - Институт государственного значения для Республики Сербия, г. Белград, Республика Сербия, корреспондент

в TU Wien, Институт инженерии информационных систем, г. Вена, Республика Австрия; Белградский университет, инженерно-строительный факультет, г. Белград, Республика Сербия

РУБРИКА ГРНТИ: 27.00.00 МАТЕМАТИКА:

27.23.25 Специальные функции,

27.25.15 Дескриптивная теория функций

ВИД СТАТЬИ: оригинальная научная статья

Резюме:

Введение/цель: Устанавлены некоторые суммы поли- логарифмической фрункции, связанные с гармоническими числами.

Методы: Подход основан на использовании методов суммирования.

Результаты: В данной статье обобщены результаты ряда дзета-функций, связанных с гармоническими числами.

Выводы: В следствие обобщения получены различные интересные ряды.

Ключевые слова: полилогарифрмическая фрункция, ряды, гармонические числа, интегрирование.

\section{ХАРМОНИЧНИ НИЗ СА ПОЛИЛОГАРИТАМСКИМ ФУНКЦИЈАМА}

Вук Стојиљковић ${ }^{\mathrm{a}}$, Никола Фабиано ${ }^{6}$, Весна Шешум Чавићв

а Универзитет у Новом Саду, Природно-математички фракултет, Нови Сад, Република Србија 
${ }^{б}$ Универзитет у Београду, Институт за нуклеарне науке "Винча"Институт од националног значаја за Републику Србију,

Београд, Република Србија, аутор за преписку

в Технички универзитет у Бечу, Институт за инжењерство информационих система, Беч, Република Аустрија; Универзитет у Београду, Грађевински факултет, Београд, Република Србија

ОБЛАСТ: математика

ВРСТА ЧЛАНКА: оригинални научни рад

\section{Сажетак:}

Увод/циљ: Установљене су неке суме полилогаритамске функције повезане са хармонијским бројевима.

Memode: Приступ се заснива на коришћењу метода сумирања.

Резултати: Генерализовани су резултати низа зета фрункција повезаних са хармонијским бројевима.

Закључак: Добијени су различити занимљиви низови као последица генерализације.

Кључне речи: полилогаритамска функција, серија, хармонијски бројеви, интеграција.

Paper received on / Дата получения работы / Датум пријема чланка: 30.11.2021. Manuscript corrections submitted on / Дата получения исправленной версии работы / Датум достављања исправки рукописа: 03.01.2022.

Paper accepted for publishing on / Дата окончательного согласования работы / Датум коначног прихватања чланка за објављивање: 04.01.2022.

() 2022 The Authors. Published by Vojnotehnički glasnik / Military Technical Courier (http://vtg.mod.gov.rs, http://втг.мо.упр.срб). This article is an open access article distributed under the terms and conditions of the Creative Commons Attribution license (http://creativecommons.org/licenses/by/3.0/rs/).

( 2022 Авторы. Опубликовано в "Военно-технический вестник / Vojnotehnički glasnik / Military Technical Courier" (http://vtg.mod.gov.rs, http://втг.мо.упр.срб). Данная статья в открытом доступе и распространяется в соответствии с лицензией "Creative Commons" (http://creativecommons.org/licenses/by/3.0/rs/).

(c) 2022 Аутори. Објавио Војнотехнички гласник / Vojnotehnički glasnik / Military Technical Courier (http://vtg.mod.gov.rs, http://втг.мо.упр.срб). Ово је чланак отвореног приступа и дистрибуира се у складу са Creative Commons лиценцом (http://creativecommons.org/licenses/by/3.0/rs/).

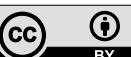

